\title{
On the use of a hexapod table to improve tumour targeting in radiation therapy
}

\author{
Jürgen Meyer ${ }^{1,2}$, Matthias Guckenberger2 ${ }^{2}$, Jürgen Wilbert ${ }^{2}$ and Kurt Baier ${ }^{2}$ \\ ${ }^{1}$ University of Canterbury, ${ }^{2}$ University of Würzburg \\ ${ }^{1}$ New Zealand, ${ }^{2}$ Germany
}

\section{Introduction}

Following the discovery of $\mathrm{x}$-rays by Röntgen at the end of the 19th century it did not take long before ionising radiation was used to treat cancer (Del Regato, 2000). Early treatments were not very accurate in terms of targeting the tumour and sparing surrounding healthy tissue. Geometrical accuracy was in the range of centimetres rather than millimetres. Since those pioneering days considerable improvements facilitated by several technological advances and treatment strategies have been made (Abrams, 1992; Schlegel et al., 2006; Webb, 2001). Today treatment with high energy ionising radiation is one of the three traditional forms of medical treatment used to treat cancer and for palliation of symptoms. It may be used alone or in conjunction with surgery or chemotherapy. It is unrivalled as a treatment in cases where surgical removal of the cancer is impossible or might debilitate the patient, e.g. tumours that are infiltrative or located close to a critical organ such as the spinal cord. The more precisely the tumour can be localised the better it can be targeted.

Improvements over the last decades in both anatomical and functional imaging as well as o detector technology have made it possible for tumours to be more accurately located (Apisarnthanarax \& Chao, 2005; Grosu et al., 2005; Jaffray \& Siewerdsen, 2000; Ling et al., . 2000). Improved localization has the potential to reduce safety margins around the tumour ㅇ volume leading to more patient-specific but also more complex shaped target volumes D reflecting the demarcation of the tumour in the medical images. Delivering the radiation $\stackrel{\Phi}{. \pm}$ dose to these irregular target volumes requires a great deal of technological but also human effort. It is against this background that radiotherapy has become a discipline with a quest for precision and sub-millimetre accuracy (Guckenberger et al., 2006a; Meyer et al., 2007; § Murphy, 1997; Solberg et al., 2004; Yu et al., 2004).

IN Considering the diversity and elasticity of a human body, its temporal biological variations Tु and various sources of organ motion this is a challenging pursuit requiring sophisticated $\widetilde{\pi}$ technology. The use of robots in radiotherapy is beginning to play an increasingly important of role in achieving this goal. The challenge is to integrate and utilize robotic technology in a \& judicious and safe way. The aim is to be able to perform treatments, which were previously 0 unattainable, less accurate and/or reliable or dependent on the skills and experience of the medical team performing the treatment.

$\underset{\mathbb{0}}{0}$ For a detailed description and review of the fundamentals of radiotherapy the reader is referred to other sources (Khan, 2003; Podgorsak, 2005). However, a brief introduction is 
given here to introduce some of the basics and in doing so the reader should become familiar with the principles of radiotherapy. Radiotherapy can be subdivided into two main categories, namely brachytherapy (Greek: brachy = short) and teletherapy (Greek: tele = long). Brachytherapy refers to therapeutic use of encapsulated (sealed) radionuclides within or close to a tumour. To achieve this, the radiation source has to be placed inside the patient where it gives a high dose of radiation to the cancerous tissue in close proximity. Due to the rapid fall-off of the intensity of the radiation, healthy tissues further away from the source receive considerably less dose. Teletherapy, on the other hand, also referred to as external beam radiotherapy (EBRT), is a more common technique for treating cancer patients. In teletherapy, high-energy photon (or electron) beams are typically applied to treat deepseated tumours. These beams, which reach megavoltage (MV) energies, are produced on medical linear accelerators (Greene \& Williams, 1997), denoted linacs. The beams are directed from the outside of the patient towards the tumour thereby depositing energy in the tissue as they penetrate through the body. By applying multiple isocentric fields from a number of different directions, the dose to the tumour can be controlled and the dose to the surrounding healthy tissues minimised. Typically, the isocentre is located in the centre of the tumour and is the pivot point of the linac to which the radiation beams are directed. The challenge with both techniques is to deliver a prescribed dose as conformal as possible to the tumour volume and at the same time keep the dose to surrounding tissues as low as one reasonably can. The treatment planning parameters, such as beam energy, beam shape, relative beam weight and beam orientation, are crucial and are optimized at the treatment planning stage. In order for the treatment to be executed adequately, the radiation has to be delivered exactly as specified in the treatment plan. In practice this is often difficult to achieve due to the flexibility and day-to-day variations in the patient's anatomy (e.g. different rectal filling, movement due to breathing, weight loss) but, with regards to teletherapy, also due to the difficulty of repositioning and aligning the patient in exactly the same position every day (Gildersleve et al., 1995; Noel et al., 1995). Inaccurate alignment of the radiation beam with the patient can lead to two things. Critical organs may receive an unwanted high dose leading to normal tissue complications and/or the tumour may receive a reduced dose leading to a loss in tumour control. Tumour control is achieved if all clonogenic tumour cells, cells that have the potential to proliferate indefinitely and give rise to a colony of cells, are inactivated. Normal tissue complications and tumour control have an impact on both the patient's quality-of-life and survival, respectively, and at all cost should be minimised and maximised, respectively. The relationship between the benefit of radiotherapy and harm due to toxicity of the healthy tissues is called therapeutic ratio. Maximizing the therapeutic ratio is one of the main goals of current research. This clearly highlights the importance of spatial accuracy in the delivery of radiotherapy. Amongst other approaches, robots have been utilized to improve accuracy and reproducibility in radiotherapy.

In the following section, an overview of the literature regarding the use of robotic systems in radiotherapy will be given. Note that these robots do not necessarily fall into the category of walking or climbing robots. This will be followed in sections 3 and 4 , respectively, by a more detailed description of the two main applications studied by the authors. They are: 1) the use of a robotic hexapod treatment table for accurate patient positioning in six degrees of freedom (DOF) and 2) the utilization of an industrial robot to develop a system to operate the robotic hexapod table dynamically with the aim to compensate for breathing induced 
tumour motion in real-time during radiotherapy treatment. The former approach has been successfully implemented into clinical routine and the clinical benefits but also the potential risks associated with this new technology will be addressed. The use of an additional robot to simulate dynamic compensation of tumour motion in real-time is an active area of research and the experimental set-up as well as encouraging preliminary results will be presented.

\section{Literature on robots in radiotherapy}

Depending on the definition of the term "robot" several computer controlled electromechanical systems conveying some sense of intelligence or agency could be referred to as robots. For brevity, only the most relevant ones guided by imaging technology are mentioned here. To improve the geometrical targeting for both teletherapy and brachytherapy the radiation source needs to be positioned accurately with respect to the identified tumour volume. Based on imaging information (or any other signal that provides relevant information) this can be done by either adjusting the radiation source relative to the patient or the patient relative to a fixed radiation source.

In brachytherapy, the radioactive sources are guided into or near the tumour through needles. A number of groups are currently working on prototypes and have demonstrated the feasibility of using robots as a moveable needle guide (Dimaio et al., 2007; Wei et al., 2004) leaving the actual insertion of the needle into the target organ to the physician. Other groups are working on placing radioactive seeds directly into the lesion, e.g. the lung (Trejos et al., 2007) or the prostate (Fichtinger et al., 2006). For all of these procedures image-guided feedback was provided by either trans-rectal ultrasound (TRUS) or by means of magnetic resonance imaging (MRI). The favourable positioning accuracy of the robotically assisted procedures was demonstrated in phantom studies. Another advantage of robotic approaches is that some of the limitations of traditional brachytherapy, such as merely parallel insertion of the needles through a template into the prostate, can be overcome. With robots, it is possible to position the needles and radioactive seeds accurately at any angle thereby making it possible to place sources into positions that were previously not attainable due to the anatomy of the patient. An example for this is pubic arch interference with the path of the needle. For parallel insertion of the needle through a template, some parts of the prostate are impossible to be targeted, whereas with a robot this is not a restraint.

Probably the most exciting development in radiotherapy with regards to robots was the development of the cyberknife by Adler at the Department of Neurosurgery at Stanford University Medical Centre (Adler et al., 1997; Chang et al., 1998). The cyberknife is a 6 MV linear accelerator mounted to a robotic manipulator. It was originally designed for treatment of cranial lesions but has been modified to treat extra-cranial tumours (Murphy et al., 2000). Guided by stereo x-ray imaging the cyberknife can deliver non-isocentric pencil beams with a high degree of accuracy from arbitrary points in space. One of the main advantages of robotic linacs in general is the fact that non-spherical target volumes can be irradiated with a higher degree of conformity than with isocentric systems (Webb, 1999; 2000). In addition, traditional frame based systems require the patient to be fixed to the treatment table for targeting and immobilisation reasons. This can be done by invasive or non-invasive means depending on the accuracy required. With the cyberknife, the patient can be on the treatment table without restraints of any kind. Movement of the patient or the tumour is 
detected by the imaging system and corrected by the cyberknife in real-time (Murphy, 2004). Currently the cyberknife is the only commercial system that can track and correct for moving objects in real-time. In section 4, an alternative approach to accomplish this on a conventional linac in combination with a hexapod table will be described.

\section{Inter-fractional set-up correction in six degrees of freedom}

In radiotherapy, the total treatment dose is usually not given in one single fraction but in a fractionated fashion over a period of four to seven weeks. Between these so-called treatment fractions, differences in the location of relevant organs and the tumour with respect to the treatment isocentre are referred to as inter-fractional set-up errors. Image-guidance provides the information for determining patient set-up errors and hence correction. In this context, image-guidance relates to images obtained immediately preceding treatment, with the patient in the treatment position. These images are compared, either manually or automatically, with initial images taken prior to treatment planning at an earlier stage. The spatial differences between planned and actual position of a structure or organ(s) of interest is referred to as patient set-up error.

Currently, it is common practice to obtain a pair of orthogonal projection portal images of the treatment region prior to treatment, using the MV therapy beam, for determining patient set-up errors. These portal images are then compared with digitally reconstructed radiographs from the initial planning computed tomography (CT) image set. The quality of the MV portal images is not sufficient to detect small differences in soft tissue contrast and hence is mainly restricted to detect bony anatomy and air cavities; the tumour itself is usually not visible in the electronic portal-imaging device (EPID) images. Image-guidance by this means is not ideal as it is well known that soft tissue tumours do not correlate well with bony anatomy (Guckenberger et al., 2006c). Kilovoltage (kV) portal imaging was then introduced and provided soft tissue contrast but it was $\mathrm{kV}$ cone-beam computed tomography (CBCT) attached to the linear accelerator that revolutionised image guidance. Whereas previously two-dimensional projections of the patient's anatomy were utilized to determine set-up errors, with CBCT it was now possible to compare full three-dimensional (3D) volumetric information with a high resolution and quality. In fact, with regards to the correction parameters it was now possible to determine set-up errors in six DOF compared to two DOF, which is seen as an enormous improvement. Initially it was not possible to correct set-up errors in six DOF due to the design of the linear accelerator treatment table. Conventional integrated treatment tables possess three axes of translational and one fixed axis of rotational movement. Although they can be moved manually or automatically (Bel et al., 2000) in translational direction they have limited accuracy (Brock et al., 2002; Sharpe et al., 2006) with respect to the resolution of the image registration. This is when the need for a more accurate treatment table arose. The first such combination of a commercial CBCT and a commercial robotic hexapod table was installed in 2005 and clinically implemented at the University of Würzburg. Since then, both units have been fully integrated and are commercially available as a package.

\subsection{System description}

An image of the set-up at the University of Würzburg is shown in Figure 1. The Elekta Synergy STM linac is equipped with two imaging systems perpendicular to each other. They 
are an EPID, referred to by the manufacturer as iviewGTTM, to obtain MV portal images of the therapy beam and a $\mathrm{kV} \mathrm{CBCT}$ unit, referred to as x-ray volume imaging (XVITM).

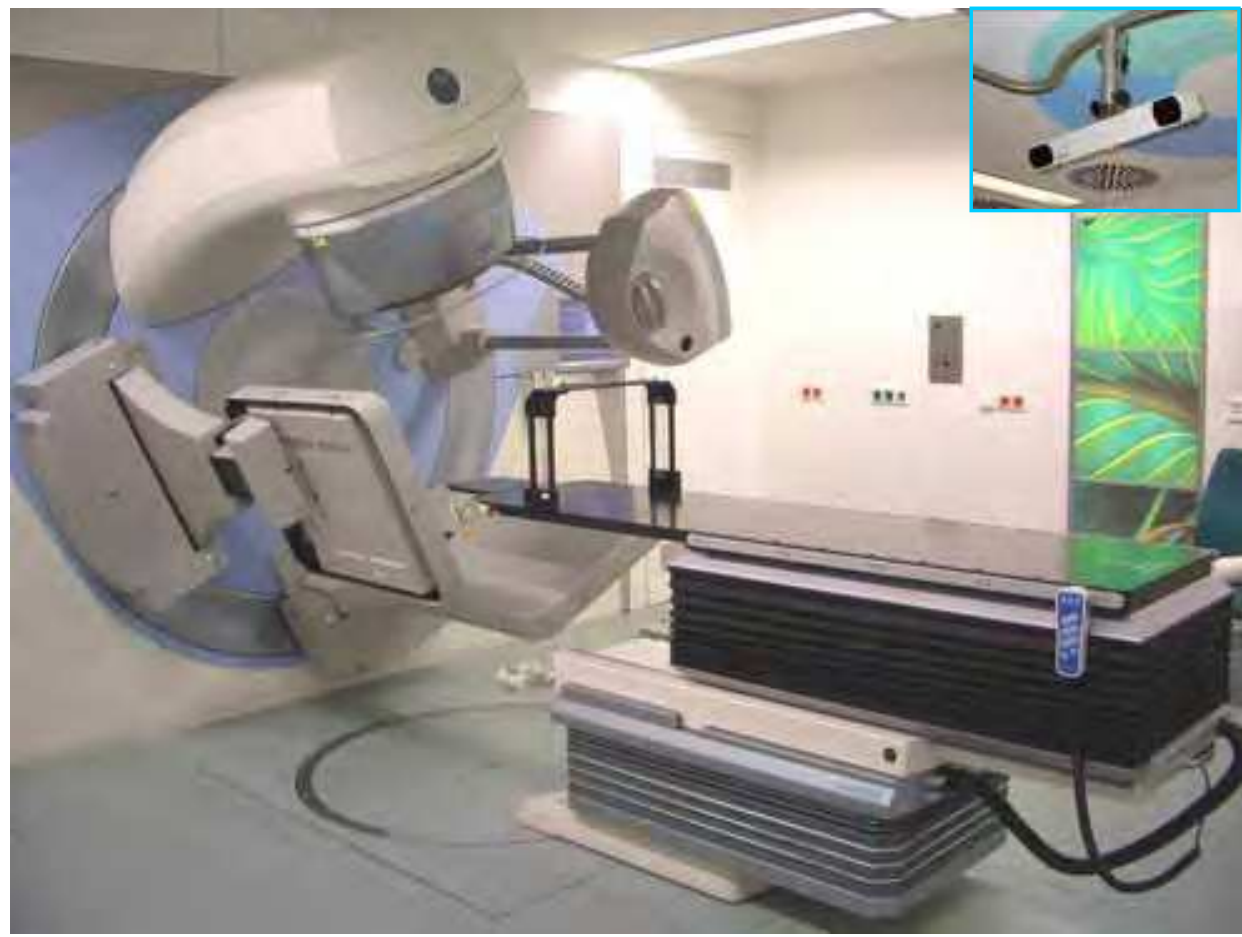

Figure 1. Linear accelerator installation at the University of Würzburg. The HexaPOD robot table is mounted on a standard treatment table. The MV treatment beam emerges through the gantry head, which in this picture is positioned at the top (ca. $\left.340^{\circ}\right)$. The MV EPID positioned opposite at the bottom (ca. $160^{\circ}$ ) records the radiation after it has passed through the patient and produces portal images. The $\mathrm{kV} \mathrm{X-ray} \mathrm{source} \mathrm{and} \mathrm{corresponding} \mathrm{flat} \mathrm{panel,}$ positioned at the right $\left(\mathrm{ca} .70^{\circ}\right)$ and left $\left(250^{\circ}\right)$, respectively, are rotated around the patient to produce cone-beam CT images. The inset on the top right depicts the tracking camera, which tracks the reflectors on the c-shaped bridge (see also Figure 2)

The Medical Intelligence HexaPODTM treatment table (Schwabmünchen, Germany) is a robotic carbon fibre table, referred to as iBeam ${ }^{\mathrm{TM}}$, with six independent actuators (Medical Intelligence, 2006). It is rigidly mounted on top of the standard treatment table. It has six DOF and can correct translational but also rotational errors. The rotational movements are denoted as pitch, roll and yaw. Table positioning is computer-controlled via a personal computer outside the treatment room. The treatment procedure is such that the patient is first aligned on the treatment table. Then the gantry is rotated around the patient and projection images are acquired with XVI at regular intervals. The projections are reconstructed and the resulting CBCT image set, or part thereof (a sub-volume), is coregistered with the planning CT. The resulting six correction parameters are then transferred to the software (iGuide ${ }^{\mathrm{TM}}$ ) that controls the HexaPOD table. Due to the nature of the HexaPOD architecture all three pairs of legs have to be moved in order to reposition the 
table appropriately along or around any of the axes. It is important that the axis of rotation of the HexaPOD table is identical with the correction reference point for the image registration. The correction reference point is the point relative to which translational and rotational errors are specified in the image registration process. If these points are not identical the order in which the translational and rotational errors are corrected matters and has an impact on the overall position accuracy (Murphy, 2007). Consequently, if they are not matching, the patient could in fact be positioned further away from the optimum position than what was intended. The HexaPOD table is fully integrated with the linac and therefore the correction reference point is known. One of the features that make the HexaPOD table particularly apt for this application is that it can rotate around any point in space with a high degree of absolute positioning accuracy. The absolute positioning accuracy is achieved by means of an infrared tracking camera (Polaris, NDI, Waterloo, Ontario, Canada) mounted to the ceiling (Figure 1). The tracking camera tracks in real-time a set of passive reflectors rigidly connected to the table top (Figure 2). This real-time feedback system provides continuous information regarding the actual position of the table in the room coordinate system. The overall accuracy of the CBCT/HexaPOD combination is, however, dependent on several factors. A study was conducted to assess the system under clinical conditions for high precision cranial stereotactic treatments.

\subsection{Experimental accuracy study}

The overall accuracy of the CBCT/HexaPOD system depends on multiple factors. The most important ones are the agreement between the $\mathrm{kV}$ beam imaging isocentre and the $\mathrm{MV}$ therapy beam isocentre of the linac, image registration accuracy, the reproducibility of the positioning of the retractable parts of the imaging system, the relationship between the coordinate systems of the linear accelerator and the infrared positioning system and the positioning accuracy of the HexaPOD table. It has been mentioned previously that high precision radiotherapy strives for sub-millimetre accuracy. This can only be achieved if all individual parts involved in the treatment set-up and correction chain work smoothly together. In Figure 2 the experimental set-up, reflecting a clinical stereotactic treatment, is shown. Instead of a patient, an anthropomorphic head phantom was utilized for this accuracy study. For this kind of treatment, the head is tightly surrounded by a scotch cast wrapping mask so that it is rigidly connected with a stereotactic head frame. This minimises patient movement but also relates to the co-ordinate system of the linac the exact location of the tumour through three-dimensional computer-aided planning software.

The accuracy of the combined system was first tested by scrutinizing the minimum achievable misalignment (set-up error) detectable by the imaging system, which includes image acquisition and image registration. Thereafter a series of predefined translational errors, a series of rotational errors and a combination of both based on data obtained from clinical treatments were automatically corrected by means of the HexaPOD table and the infrared camera system. A detailed description of the experimental procedure and results are described by Meyer et al. (Meyer et al., 2007). It was found that the system performance of the imaging system alone was very stable with mean translational and rotational errors of below $0.2 \mathrm{~mm}$ and below $0.2^{\circ}$, respectively. The integration of the HexaPOD table in terms of overall positioning accuracy was similar with mean translational errors of below $0.3 \mathrm{~mm}$ and mean rotational errors of below $0.3^{\circ}$. These results indicate that it is indeed possible to achieve an overall position accuracy and reproducibility in a phantom in the order of tens of 
millimetres. Note that the errors relate to the inherent capability of the technology and a patient might add some additional uncertainty.

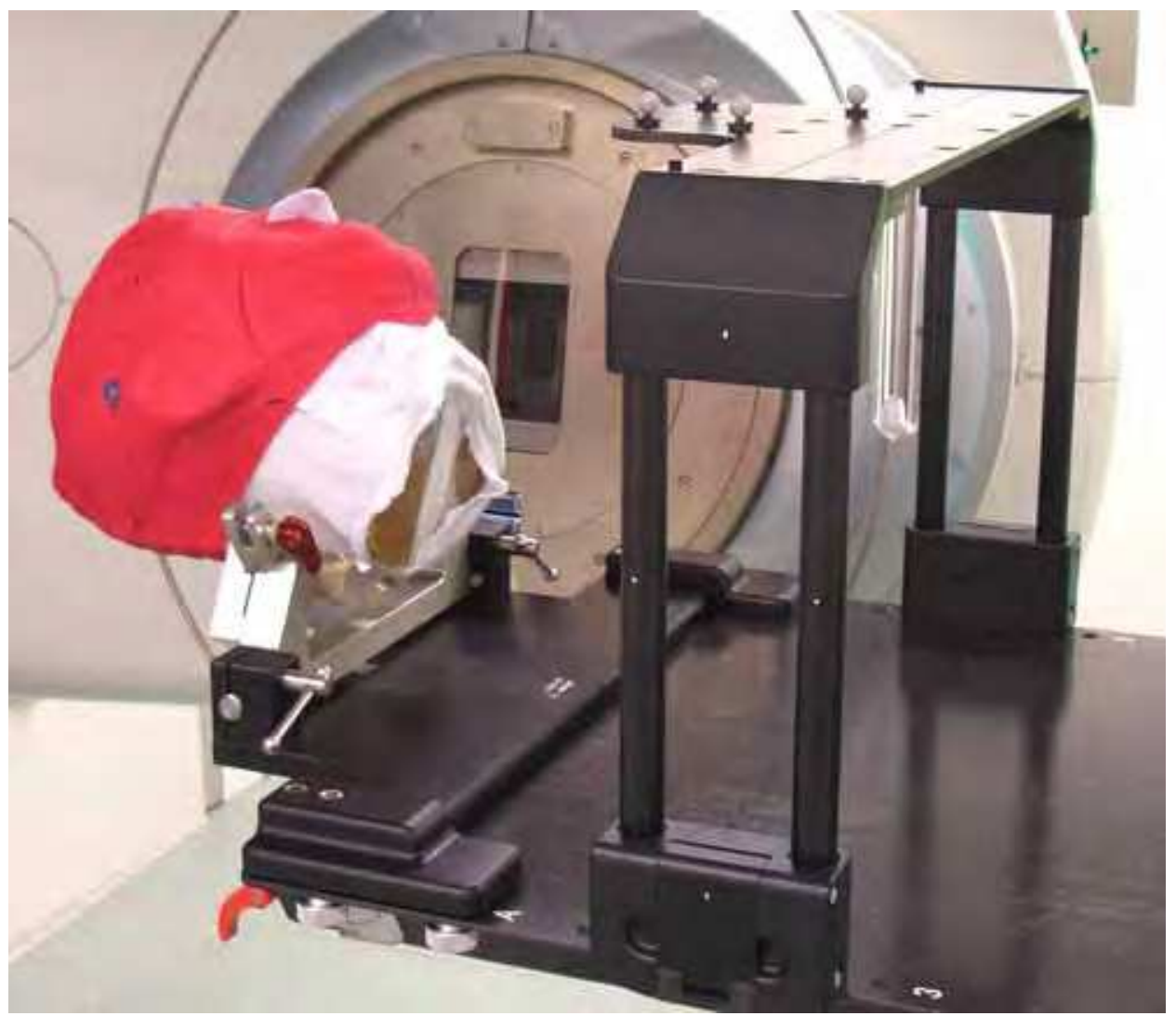

Figure 2. Experimental set-up. Head phantom in a stereotactic head frame attached to the HexaPOD table. The reflectors on the bridge connected to the HexaPOD are tracked by the tracking camera (see inset Figure 1) for accurate absolute positioning

Care has to be taken, not to be tempted to correct rotational set-up errors for every treatment. For most standard clinical situations, there might not be a noticeable benefit, especially when the tumour volumes are more or less spherical. In fact, it was found that correcting rotational errors for patients who are not fixed in a mask or body frame could lead to substantial inaccuracies. This is because patients tend to involuntarily counter react to table tilt and therefore some of the anatomy might become deformed due to involuntary muscular activity (Guckenberger et al., 2007; Schweikard et al., 2000). For high precision stereotactic treatments were patients are appropriately immobilised this effect was not observed (Guckenberger et al., 2006a; Guckenberger et al., 2007). On the other hand, for treatments such as in the brain, where lesions with dimensions of a few millimetres are adjacent to vital structures, sub-millimetre accuracy can make a difference. This is also true for paraspinal tumours (Greek: para $=$ near to) wrapped around the spinal cord (Guckenberger et al., 2006b). 


\section{Dynamic compensation for intra-fractional tumour movement}

In the previous section only correction of set-up errors between treatment fractions were considered. Some tumours, however, move periodically during each single treatment session. Tumours in the lung, breast or liver often exhibit this property and displacements of up to $3 \mathrm{~cm}$ have been reported (Engelsman et al., 2005; Seppenwoolde et al., 2002). To account for this, large margins are required to ensure that the tumour is in the beam at all times. Consequently, large radiation fields are necessary. This is at cost of the healthy tissues in the beam path, which, inevitably, receive a large radiation dose. Confining the treatment beams to the immediate surroundings of the tumour volume, i.e. using small margins, would reduce the normal tissue complications and enable dose escalation to the tumour. It is expected that such dose escalation would translate into higher rates of tumour control and improve survival of the patients. Several strategies are possible to manage tumours that move during irradiation. One possibility is to switch the beam on every time the tumour is in a certain position or phase and switch it off for the rest of the cycle. This is referred to as gated radiotherapy (Keall et al., 2002; Kubo \& Hill, 1996). Although this approach has been applied clinically, it has several disadvantages, such as prolonged treatment time. Of more concern is the fact that the beam is triggered by the breathing signal and often there is no real-time verification of the actual tumour position during beam-on time. However, it has been shown that it is achievable using implanted markers (Berbeco et al., 2005b; Keall et al., 2004). Another approach, to be discussed now, is tracking the tumour during irradiation and correcting for it in real-time (Keall et al., 2001; Murphy, 2004). Corrections for tumour motion can be applied by either dynamically adjusting the aperture of the radiation beam shaping device (Keall et al., 2006), the multi-leaf collimator (MLC), or by moving the patient relative to the stationary radiation beam (D'Souza et al., 2005). The latter can be achieved by means of the HexaPOD treatment table and will be discussed henceforth. The idea is to counter-steer the tumour movement dynamically with the HexaPOD table. With this approach, the tumour becomes fixed in space such that it appears almost stationary in the beams-eye-view of the accelerator. This allows a reduction of the radiation fields leading to better protection of the surrounding tissue. The system with which dynamic compensation of tumour motion is accomplished is the so-called Würzburg Adaptive Tumour Tracking System (WATTS). The main components of WATTS are based on the same equipment mentioned previously, i.e. the linac and the hexapod robot table. The prototype system currently being developed is comprised of additional features enabling tracking and correction of tumour movement.

\subsection{Würzburg Adaptive Tumour Tracking System (WATTS)}

An overview of the components of WATTS is depicted in Figure 3. The system consists of the Elekta Synergy S linac, the HexaPOD, optional add-on devices, such as a tool with infrared reflectors, and in-house hard and software solutions. The basic principle is as follows: information regarding the tumour position is obtained in real-time by two independent means, namely MV imaging (lower branch) and tracking of an infrared reflector tool placed on the abdomen of the patient (upper branch). The idea is to have two independent data sets available that provide excess information regarding the tumour position. This is to ensure that the tumour position can be determined safely even when one of the systems temporarily fails to provide reliable data. By determining and tracking the tumour position in each frame the magnitude and frequency of the tumour movement may 
be determined. Due to the nature of these images, it is a difficult task due to inherently poor soft tissue contrast. In a feasibility study it was demonstrated that it is nevertheless possible to track moving objects, using appropriate algorithms, even in noisy portal images (Meyer et al., 2006b). This has been implemented into the in-house software PortalTrack (PC1). It is nonetheless not prudent to merely rely on portal imaging information since in certain situations the exact tumour position might be impossible to be unambiguously determined. The second data set is based on the movement of the tool with reflectors placed on the abdomen of the patient.

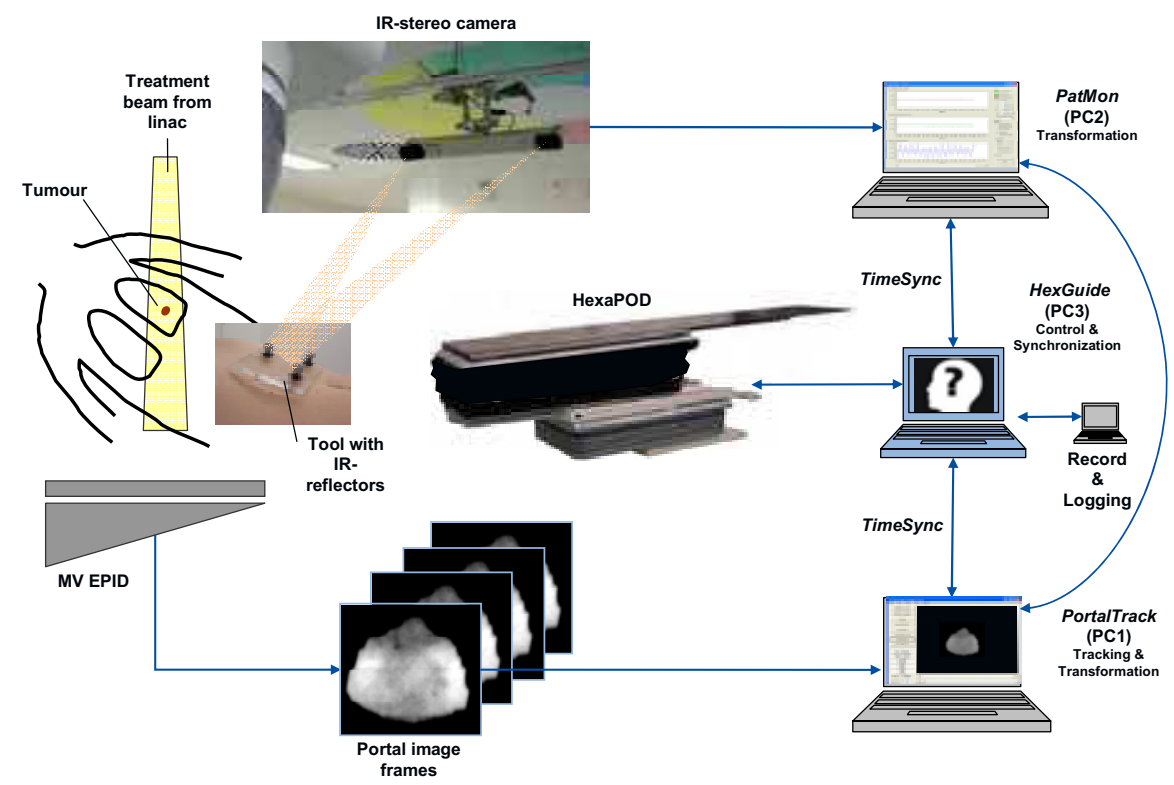

Figure 3. Overview of the "Würzburg Adaptive Tumour Tracking System" (WATTS)

This tool is tracked simultaneously with the same tracking camera as the HexaPOD table. Note that the infrared camera is able to track multiple tools at the same time. The respiratory signals are processed and transformed into the co-ordinate system of the linac in the in-house software PatMon (PC2). Both PortalTrack and PatMon send the data to the control computer HexGuide (PC3), where the necessary table movements are calculated. Due to the time delay between determining the tumour position and actual correction with the HexaPOD table, it is crucial to predict the tumour position ahead of time. Theoretically, both signals could be used independently as the basis for prediction. Because the respiratory signal is acquired at a more than 10 fold rate than the MV images it will be used primarily for prediction of the tumour trajectory (Meyer et al., 2006a). The signal from PortalTrack provides a means to verify the applied correction and to adapt the prediction model if necessary.

During the ongoing development stage of WATTS it is essential to vigorously test the system behaviour under realistic conditions. This will help to determine the potentials but also limitations of WATTS. This can only be accomplished by imitating tumour movement at different speeds and amplitudes under controlled setting and assessing how well the system can compensate in extreme situations. 


\subsection{Experimental study}

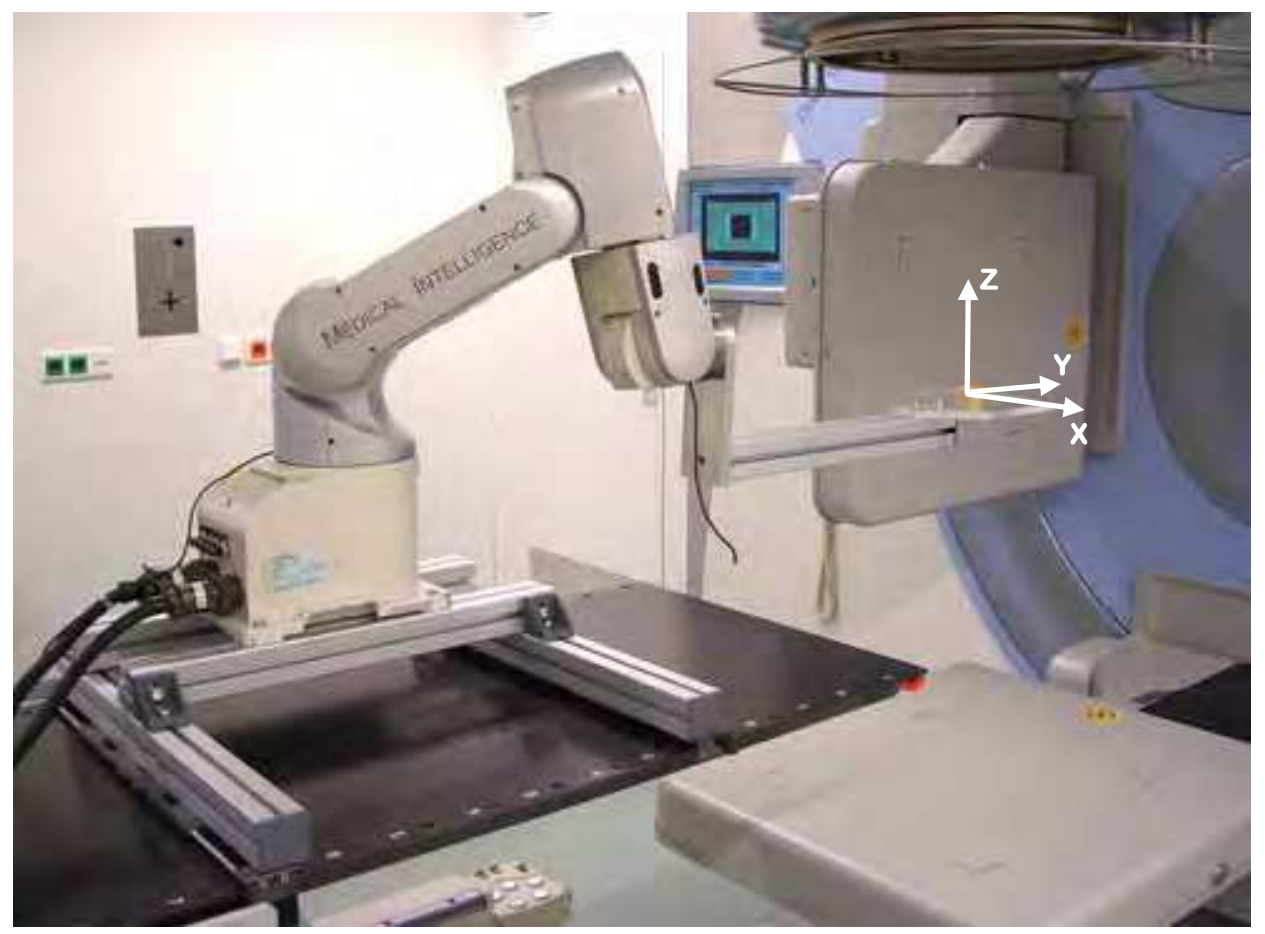

Figure 4. Six-axis robot mounted on the HexaPOD robot table. The gantry head is at the top $\left(0^{\circ}\right)$ and the MV EPID is at the bottom $\left(180^{\circ}\right)$. Attached to the robot is a phantom with a wooden disk on a slab of Plexiglas representing the object to be tracked. Note that for actual treatment of patients a head extension board is attached to the HexaPOD table

To simulate tumour trajectories an industrial six-axis robot (MELFA Industrial Robot, RV1A Series, Mitsubishi Electric, Ratingen, Germany) was mounted on the HexaPOD table (Figure 4). An attachment was constructed to affix a phantom to the robotic arm. The phantom consisted of a wooden disk, representing the tumour, placed on a slab of Perspex. The robot was positioned such that translational movements along the three main axes were possible along a straight path ( $x, y$ and $z)$. To test the system behaviour of WATTS, triangular trajectories of different magnitude and speed were fed to the six-axis robot. The direction of movement was along the $\mathrm{x}$ and $\mathrm{y}$-plane. For this experimental set-up, a reflector

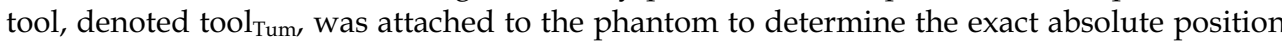
of the tumour model in the room coordinate system. Note that for application on a patient the tumour position would have to be devised from both the respiratory signal and the portal image information instead. With this approach, there are virtually no uncertainties in the exact tumour location for testing the dynamic behaviour of the HexaPOD table. Since the tumour model was connected to the HexaPOD through the six-axis robot, tool Tum $_{\text {Tecords }}$ the tumour movement with the HexaPOD movement superimposed. In order to determine the relative movement of the tumour model with respect to the HexaPOD the reflectors on the c-shaped bridge, denoted tool $_{\mathrm{Hex}}$, were tracked simultaneously. During playback of the 
trajectories, the positions of the tumour, i.e. tool $_{T u m}$ and the table, i.e. tool $\mathrm{H}_{\mathrm{Hex}}$, were acquired at a rate of approximately $40 \mathrm{~Hz}$ using the tracking camera and PatMon. Based on the difference of the two signals, which is equivalent to the tumour motion relative to the table, the position of the HexaPOD table was corrected (HexGuide) in real-time (cf. Figure 3). In other words, a movement by the six-axis robot in e.g. +y-direction was compensated by a counter movement in -y-direction by the HexaPOD and vice versa in $x$-direction. With this arrangement, continuous movement of the six-axis robot was counteracted continuously by the HexaPOD table resulting in a reduced tumour motion in the treatment room space.

\subsection{Preliminary results}
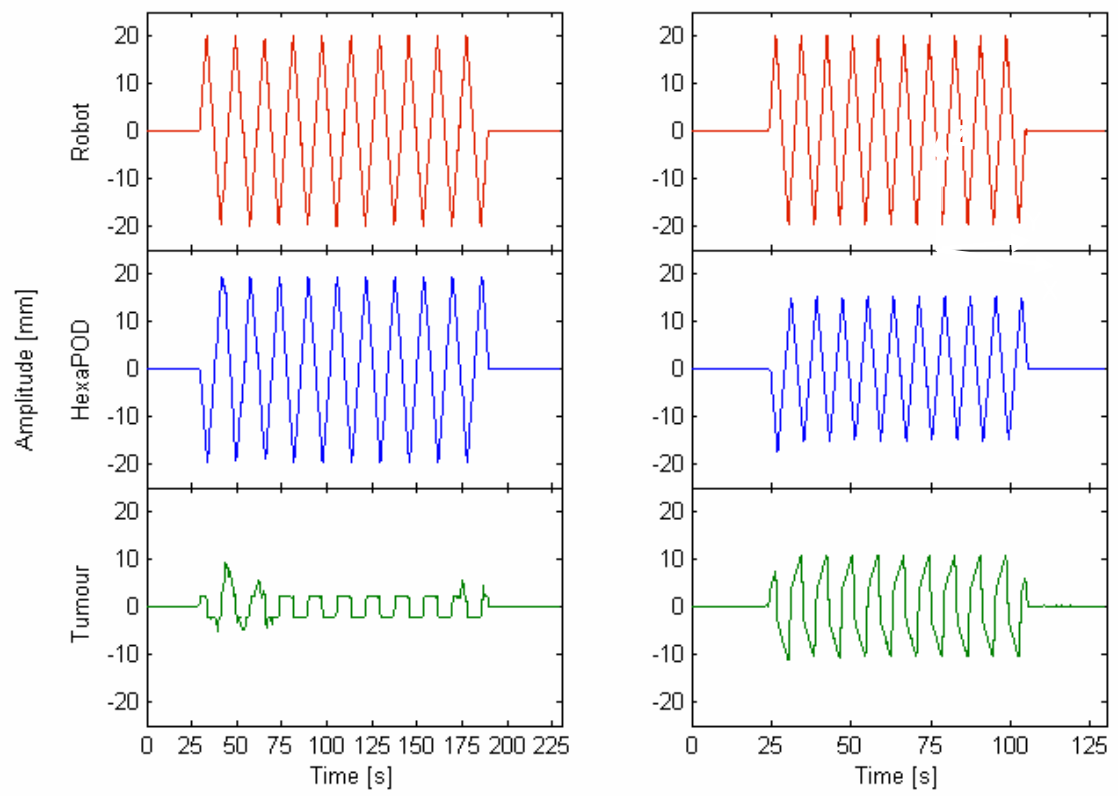

Figure 5. Trajectories for robot motion with a maximum speed of $5 \mathrm{~mm} / \mathrm{s}$ (left) and $10 \mathrm{~mm} / \mathrm{s}$ (right). The HexaPOD data represents the table movement applied by HexGuide to countersteer tumour motion. The residual tumour motion, with respect to the treatment beam, is the difference between robot and HexaPOD movement

The table corrections determined by HexGuide were based on the actual PatMon signals only; no prediction of future positions was used for this initial experimental study. In Figure 5 , the trajectories for the robot, representing the tumour model, the HexaPOD and the residual tumour motion are depicted. The amplitude of the robot movement was $\pm 20 \mathrm{~mm}$ with a maximum speed of $5 \mathrm{~mm} / \mathrm{s}$ and $10 \mathrm{~mm} / \mathrm{s}$, respectively. It can be seen that in both cases the movement of the table was inversely phased to the robot motion. This results in a clearly reduced residual motion of the tumour. The noticeable irregularities in the graphs stem from gaps in the PatMon data. Apart from these irregularities, the resulting absolute tumour movement was decreased to maximum errors of approximately $\pm 2 \mathrm{~mm}$ and $\pm 10 \mathrm{~mm}$ for maximum tumour speeds of $5 \mathrm{~mm} / \mathrm{s}$ and $10 \mathrm{~mm} / \mathrm{s}$, respectively. It is expected that more 
realistic sinusoidal tumour trajectories would result in even smaller overall errors. The sudden changes in the triangular tumour trajectories require the HexaPOD to accelerate and decelerate rapidly. As can be seen in Figure 5, this constitutes the main source of error for the residual tumour motion. Furthermore, given the fact that no efforts were made to compensate for delay in the control system this is a promising result. The next step will be to implement predictive models to determine tumour positions ahead of time with the overall aim to decrease absolute tumour movement to $1-2 \mathrm{~mm}$ for tumour speeds of up to $2-3 \mathrm{~cm} / \mathrm{s}$. If attainable, this would pave the way for smaller radiation fields resulting in less irradiation of healthy tissue.

\section{Discussion \& Conclusion}

High precision image-guided radiotherapy demands a high degree of spatial accuracy for treatment delivery. Recent advances in imaging technology for radiotherapy have been shown to yield information with respect to the tumour position with sub-millimetre accuracy. Differences between planned and actual tumour position at treatment can be calculated in six DOF. With standard equipment, it is not possible to correct for these set-up errors with the same accuracy as they were detected. Furthermore, it is not possible to correct for all rotational errors. These limitations can be overcome with the robotic HexaPOD table. With the combination of 3D volume imaging on the linac and the HexaPOD table, an overall mean positioning accuracy of $0.3 \mathrm{~mm}$ and $0.3^{\circ}$ was obtained in an experimental accuracy study. With this combination, it is possible to deliver treatment with a high degree of accuracy guided by imaging technology. This, in turn, has the potential to reduce safety margins around the tumours and hence to improve treatment outcomes. At the University of Würzburg, 6D patient set-up correction is routinely carried out for highprecision radiotherapy. Patients who most benefit from such high accuracy are those with tumours in very close proximity to critical structures, such as lesions in the brain or paraspinal lesions. The latter are frequently wrapped around the spinal cord. Due to the elongated dimensions of paraspinal tumours surrounding a critical structure, this constellation is very sensitive to rotational set-up errors, which can be corrected adequately with the HexaPOD table. It was found that although rotational errors can be corrected it is essential to ensure that the patient is appropriately immobilized. Clinical application of $6 \mathrm{D}$ position correction has revealed that several non-immobilized patients who received standard treatment in the pelvic region moved after rotational errors had been corrected. This was most likely to counter act table rotations around the patient axis. Some patients ended up with set-up errors larger than before correction. This fact highlights the fine line between the benefits and detrimental effects with this new technology. A critical evaluation is indispensable to determine the exact circumstances under which a treatment can actually be improved. At the University of Würzburg, a reasonable number of studies have been carried out to integrate image-guided radiotherapy and corrections with the HexaPOD table seamlessly into clinical routine. Today, for high-precision radiotherapy at the University of Würzburg, the HexaPOD table is an integral part of the treatment.

An active area of research is dynamic real-time compensation of tumour movement by means of WATTS. The approach described is a feasibility study. So far, its achievability has been demonstrated under non-clinical settings in a clinical environment. Although the preliminary results are very encouraging, it is still a long way from clinical implementation. Hitherto, both tracking the tumour in real-time using MV portal imaging and recording the 
breathing motion by means of the tracking camera have been applied passively to a few dozens patients. This was done without altering the actual treatment. It is envisaged that one of the first applications of WATTS will not be to compensate for tumour movement dynamically but rather to detect any drift in the tumour mean position, so-called base-line drifts, during standard lung treatments. Thus, rather then adapting the treatment WATTS may initially be used for verification purposes. This is in-line with other researchers who are working on imaging approaches to verify gated radiotherapy non-invasively (Berbeco et al., 2005a; Korreman et al., 2006). After enough confidence has been gained, one could introduce single table corrections for drifts over a certain threshold value of the mean tumour position. For gated treatments, this correction could be applied when the beam is switched off. Over time, the threshold value could be reduced so that more corrections are performed with the HexaPOD table. Finally, before treatment margins are to be reduced, the data have to be critically evaluated. Only if a benefit can be demonstrated one should consider dynamic correction of tumour motion. It is essential that the final goal of all technology in medical practice is to improve treatment results. The technology itself does not justify its use, only a benefit to the patient does.

\section{Acknowledgements}

This work was supported in part by the Bayerische Forschungsstiftung (BFS), Munich, Germany and by Elekta Oncology Systems, Crawley, UK. WATTS is a collaborative project between the Department of Radiotherapy and the Department of Computer Science VII, University of Würzburg, Germany, and Medical Intelligence $\mathrm{GmbH}$, Schwabmünchen, Germany.

\section{References}

Abrams, R.A. (1992). Recent developments in radiotherapy. Curr Opin Oncol; Vol. 4, No. 6, pp. 1099-1107.

Adler, J.R., Jr., Chang, S.D., Murphy, M.J., Doty, J., Geis, P., et al. (1997). The Cyberknife: a frameless robotic system for radiosurgery. Stereotact Funct Neurosurg; Vol. 69, No. 14 Pt 2, pp. 124-128.

Apisarnthanarax, S. \& Chao, K.S. (2005). Current imaging paradigms in radiation oncology. Radiat Res; Vol. 163, No. 1, pp. 1-25.

Bel, A., Petrascu, O., Van de Vondel, I., Coppens, L., Linthout, N., et al. (2000). A computerized remote table control for fast on-line patient repositioning: implementation and clinical feasibility. Med Phys; Vol. 27, No. 2, pp. 354-358.

Berbeco, R.I., Mostafavi, H., Sharp, G.C. \& Jiang, S.B. (2005a). Towards fluoroscopic respiratory gating for lung tumours without radiopaque markers. Phys Med Biol; Vol. 50, No. 19, pp. 4481-4490.

Berbeco, R.I., Neicu, T., Rietzel, E., Chen, G.T. \& Jiang, S.B. (2005b). A technique for respiratory-gated radiotherapy treatment verification with an EPID in cine mode. Phys Med Biol; Vol. 50, No. 16, pp. 3669-3679.

Brock, K.K., McShan, D.L. \& Balter, J.M. (2002). A comparison of computer-controlled versus manual on-line patient setup adjustment. J Appl Clin Med Phys; Vol. 3, No. 3, pp. 241-247. 
Chang, S.D., Murphy, M., Geis, P., Martin, D.P., Hancock, S.L., et al. (1998). Clinical experience with image-guided robotic radiosurgery (the Cyberknife) in the treatment of brain and spinal cord tumors. Neurol Med Chir; Vol. 38, No. 11, pp. 780783.

D'Souza, W.D., Naqvi, S.A. \& Yu, C.X. (2005). Real-time intra-fraction-motion tracking using the treatment couch: a feasibility study. Phys Med Biol; Vol. 50, No. 17, pp. 40214033.

Del Regato, J.A. (2000). One hundred years of radiation oncology, In: J. S. Tobias and P. R. M. Thomas (Ed.), Chapter 1, Oxford University Press, ISBN: 978-0-340-61387-0, New York.

Dimaio, S.P., Pieper, S., Chinzei, K., Hata, N., Haker, S.J., et al. (2007). Robot-assisted needle placement in open MRI: System architecture, integration and validation. Comput Aided Surg; Vol. 12, No. 1, pp. 15-24.

Engelsman, M., Sharp, G.C., Bortfeld, T., Onimaru, R. \& Shirato, H. (2005). How much margin reduction is possible through gating or breath hold? Phys Med Biol; Vol. 50, No. 3, pp. 477-490.

Fichtinger, G., Burdette, E.C., Tanacs, A., Patriciu, A., Mazilu, D., et al. (2006). Robotically assisted prostate brachytherapy with transrectal ultrasound guidance--Phantom experiments. Brachytherapy; Vol. 5, No. 1, pp. 14-26.

Gildersleve, J., Dearnaley, D.P., Evans, P.M. \& Swindell, W. (1995). Reproducibility of patient positioning during routine radiotherapy, as assessed by an integrated megavoltage imaging system. Radiother Oncol; Vol. 35, No. 2, pp. 151-160.

Greene, D. \& Williams, P.C. (1997). Linear Accelerators for Radiation Therapy, Taylor \& Francis Group, ISBN: 0750304022, New York.

Grosu, A.L., Piert, M., Weber, W.A., Jeremic, B., Picchio, M., et al. (2005). Positron emission tomography for radiation treatment planning. Strahlenther Onkol; Vol. 181, No. 8, pp. 483-499.

Guckenberger, M., Meyer, J., Vordermark, D., Baier, K., Wilbert, J., et al. (2006a). Magnitude and clinical relevance of translational and rotational patient setup errors: a conebeam CT study. Int J Radiat Oncol Biol Phys; Vol. 65, No. 3, pp. 934-942.

Guckenberger, M., Meyer, J., Wilbert, J., Baier, K. \& Flentje, M. (2006b). Dose escalation in treatment of (para-) spinal tumors: a novel image-guided radiation therapy (IGRT) protocol. Proceedings of 27. Deutscher Krebskongress, Berlin, Germany

Guckenberger, M., Meyer, J., Wilbert, J., Baier, K., Mueller, G., et al. (2006c). Cone-beam CT based image-guidance for extracranial stereotactic radiotherapy of intrapulmonary tumors. Acta Oncol; Vol. 45, No. 7, pp. 897-906.

Guckenberger, M., Meyer, J., Wilbert, J., Baier, K., Sauer, O., et al. (2007). Precision of ImageGuided Radiotherapy (IGRT) in Six Degrees of Freedom and Limitations in Clinical Practice. Strahlenther Onkol; Vol. 183, No. 6, pp. 307-313.

Jaffray, D.A. \& Siewerdsen, J.H. (2000). Cone-beam computed tomography with a flat-panel imager: initial performance characterization. Med Phys; Vol. 27, No. 6, pp. 13111323.

Keall, P.J., Cattell, H., Pokhrel, D., Dieterich, S., Wong, K.H., et al. (2006). Geometric accuracy of a real-time target tracking system with dynamic multileaf collimator tracking system. Int J Radiat Oncol Biol Phys; Vol. 65, No. 5, pp. 1579-1584. 
Keall, P.J., Kini, V.R., Vedam, S.S. \& Mohan, R. (2001). Motion adaptive x-ray therapy: a feasibility study. Phys Med Biol; Vol. 46, No. 1, pp. 1-10.

Keall, P.J., Kini, V.R., Vedam, S.S. \& Mohan, R. (2002). Potential radiotherapy improvements with respiratory gating. Australas Phys Eng Sci Med; Vol. 25, No. 1, pp. 1-6.

Keall, P.J., Todor, A.D., Vedam, S.S., Bartee, C.L., Siebers, J.V., et al. (2004). On the use of EPID-based implanted marker tracking for 4D radiotherapy. Med Phys; Vol. 31, No. 12, pp. 3492-3499.

Khan, F.M. (2003). The Physics of Radiation Therapy, Lippincott Williams \& Wilkins, ISBN: 0781730651,

Korreman, S., Mostafavi, H., Le, Q.T. \& Boyer, A. (2006). Comparison of respiratory surrogates for gated lung radiotherapy without internal fiducials. Acta Oncol; Vol. 45, No. 7, pp. 935-942.

Kubo, H.D. \& Hill, B.C. (1996). Respiration gated radiotherapy treatment: a technical study. Phys Med Biol; Vol. 41, No. 1, pp. 83-91.

Ling, C.C., Humm, J., Larson, S., Amols, H., Fuks, Z., et al. (2000). Towards multidimensional radiotherapy (MD-CRT): biological imaging and biological conformality. Int J Radiat Oncol Biol Phys; Vol. 47, No. 3, pp. 551-560.

Medical Intelligence. HexaPOD RT System, (2006), http:/ / www.medint.de, (06.06.2007)

Meyer, J., Baier, K., Wilbert, J., Guckenberger, M., Richter, A., et al. (2006a). Threedimensional spatial modelling of the correlation between abdominal motion and lung tumour motion with breathing. Acta Oncol; Vol. 45, No. 7, pp. 923-934.

Meyer, J., Richter, A., Baier, K., Wilbert, J., Guckenberger, M., et al. (2006b). Tracking moving objects with megavoltage portal imaging: a feasibility study. Med Phys; Vol. 33, No. 5, pp. 1275-1280.

Meyer, J., Wilbert, J., Baier, K., Guckenberger, M., Richter, A., et al. (2007). Positioning accuracy of cone-beam computed tomography in combination with a HexaPOD robot treatment table. Int J Radiat Oncol Biol Phys; Vol. 67, No. 4, pp. 1220-1228.

Murphy, M.J. (1997). An automatic six-degree-of-freedom image registration algorithm for image-guided frameless stereotaxic radiosurgery. Med Phys; Vol. 24, No. 6, pp. 857866.

Murphy, M.J. (2004). Tracking moving organs in real time. Semin Radiat Oncol; Vol. 14, No. 1, pp. $91-100$.

Murphy, M.J. (2007). Image-guided patient positioning: If one cannot correct for rotational offsets in external-beam radiotherapy setup, how should rotational offsets be managed? Med Phys; Vol. 34, No. 6, pp. 1880-1883.

Murphy, M.J., Adler, J.R., Jr., Bodduluri, M., Dooley, J., Forster, K., et al. (2000). Imageguided radiosurgery for the spine and pancreas. Comput Aided Surg; Vol. 5, No. 4, pp. 278-288.

Noel, A., Aletti, P., Bey, P. \& Malissard, L. (1995). Detection of errors in individual patients in radiotherapy by systematic in vivo dosimetry. Radiother Oncol; Vol. 34, No. 2, pp. 144-151.

Podgorsak, E.B. (2005). Radiation Oncology Physics: A handbook for teachers and students, International Atomic Energy Agency, ISBN: 92-0-107304-6, Vienna.

Schlegel, W., Bortfeld, T. \& Grosu, A.L. (2006). New Technologies in Radiation Oncology, Springer, ISBN: 3540003215, Berlin, Heidelberg, New York. 
Schweikard, A., Glosser, G., Bodduluri, M., Murphy, M.J. \& Adler, J.R. (2000). Robotic motion compensation for respiratory movement during radiosurgery. Comput Aided Surg; Vol. 5, No. 4, pp. 263-277.

Seppenwoolde, Y., Shirato, H., Kitamura, K., Shimizu, S., van Herk, M., et al. (2002). Precise and real-time measurement of 3D tumor motion in lung due to breathing and heartbeat, measured during radiotherapy. Int J Radiat Oncol Biol Phys; Vol. 53, No. 4, pp. 822-834.

Sharpe, M.B., Moseley, D.J., Purdie, T.G., Islam, M., Siewerdsen, J.H., et al. (2006). The stability of mechanical calibration for a $\mathrm{kV}$ cone beam computed tomography system integrated with linear accelerator. Med Phys; Vol. 33, No. 1, pp. 136-144.

Solberg, T.D., Goetsch, S.J., Selch, M.T., Melega, W., Lacan, G., et al. (2004). Functional stereotactic radiosurgery involving a dedicated linear accelerator and gamma unit: a comparison study. J Neurosurg; Vol. 101 Suppl 3, No. pp. 373-380.

Trejos, A.L., Lin, A.W., Pytel, M.P., Patel, R.V. \& Malthaner, R.A. (2007). Robot-assisted minimally invasive lung brachytherapy. Int J Med Robot; Vol. 3, No. pp. 41-51.

Webb, S. (1999). Conformal intensity-modulated radiotherapy (IMRT) delivered by robotic linac--testing IMRT to the limit? Phys Med Biol; Vol. 44, No. 7, pp. 1639-1654.

Webb, S. (2000). Conformal intensity-modulated radiotherapy (IMRT) delivered by robotic linac--conformality versus efficiency of dose delivery. Phys Med Biol; Vol. 45, No. 7, pp. $1715-1730$

Webb, S. (2001). Intensity-Modulated Radiation Therapy, Taylor \& Francis, ISBN: 0750306998, London.

Wei, Z., Wan, G., Gardi, L., Mills, G., Downey, D., et al. (2004). Robot-assisted 3D-TRUS guided prostate brachytherapy: system integration and validation. Med Phys; Vol. 31, No. 3, pp. 539-548.

Yu, C., Main, W., Taylor, D., Kuduvalli, G., Apuzzo, M.L., et al. (2004). An anthropomorphic phantom study of the accuracy of Cyberknife spinal radiosurgery. Neurosurgery; Vol. 55, No. 5, pp. 1138-1149. 


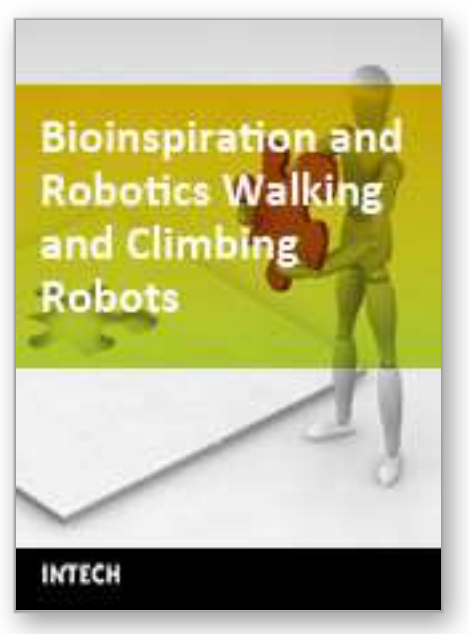

\section{Bioinspiration and Robotics Walking and Climbing Robots \\ Edited by Maki K. Habib}

ISBN 978-3-902613-15-8

Hard cover, 544 pages

Publisher I-Tech Education and Publishing

Published online 01, September, 2007

Published in print edition September, 2007

Nature has always been a source of inspiration and ideas for the robotics community. New solutions and technologies are required and hence this book is coming out to address and deal with the main challenges facing walking and climbing robots, and contributes with innovative solutions, designs, technologies and techniques. This book reports on the state of the art research and development findings and results. The content of the book has been structured into 5 technical research sections with total of 30 chapters written by well recognized researchers worldwide.

\section{How to reference}

In order to correctly reference this scholarly work, feel free to copy and paste the following:

Juergen Meyer, Matthias Guckenberger, Juergen Wilbert and Kurt Baier (2007). On the Use of a Hexapod Table to Improve Tumour Targeting in Radiation Therapy, Bioinspiration and Robotics Walking and Climbing Robots, Maki K. Habib (Ed.), ISBN: 978-3-902613-15-8, InTech, Available from:

http://www.intechopen.com/books/bioinspiration_and_robotics_walking_and_climbing_robots/on_the_use_of_ a_hexapod_table_to_improve_tumour_targeting_in_radiation_therapy

\section{INTECH}

open science | open minds

\author{
InTech Europe \\ University Campus STeP Ri \\ Slavka Krautzeka 83/A \\ 51000 Rijeka, Croatia \\ Phone: +385 (51) 770447 \\ Fax: +385 (51) 686166 \\ www.intechopen.com
}

\author{
InTech China \\ Unit 405, Office Block, Hotel Equatorial Shanghai \\ No.65, Yan An Road (West), Shanghai, 200040, China \\ 中国上海市延安西路65号上海国际贵都大饭店办公楼 405 单元 \\ Phone: +86-21-62489820 \\ Fax: $+86-21-62489821$
}


(C) 2007 The Author(s). Licensee IntechOpen. This chapter is distributed under the terms of the Creative Commons Attribution-NonCommercial-ShareAlike-3.0 License, which permits use, distribution and reproduction for non-commercial purposes, provided the original is properly cited and derivative works building on this content are distributed under the same license. 